\title{
Impaired Motor Recycling during Action Selection in Parkinson's Disease
}

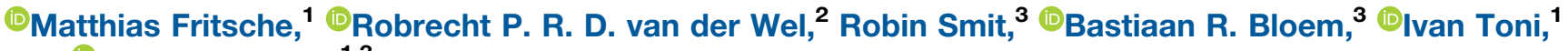 \\ and ${ }^{\circ}$ Rick C. Helmich ${ }^{1,3}$
}

https://doi.org/10.1523/ENEURO.0492-19.2020

${ }^{1}$ Centre for Cognitive Neuroimaging, Donders Institute for Brain, Cognition and Behaviour, Radboud University, Nijmegen 6525 EN, The Netherlands, '2Department of Psychology, Rutgers University, Camden, NJ 08102, and ${ }^{3}$ Donders Institute for Brain, Cognition and Behaviour; Department of Neurology; Center of Expertise for Parkinson \& Movement Disorders, Radboud University Medical Center, Nijmegen 6525 GC, The Netherlands

\begin{abstract}
Behavioral studies have shown that the human motor system recycles motor parameters of previous actions, such as movement amplitude, when programming new actions. Shifting motor plans toward a new action forms a particularly severe problem for patients with Parkinson's disease (PD), a disorder that, in its early stage, is dominated by basal ganglia dysfunction. Here, we test whether this action selection deficit in Parkinson's patients arises from an impaired ability to recycle motor parameters shared across subsequent actions. Parkinson's patients off dopaminergic medication $(n=16)$ and matched healthy controls $(n=16)$ performed a task that involved moving a handheld dowel over an obstacle in the context of a sequence of aiming movements. Consistent with previous research, healthy participants continued making unnecessarily large hand movements after clearing the obstacle (defined as "hand path priming effect"), even after switching movements between hands. In contrast, Parkinson's patients showed a reduced hand path priming effect, i.e., they performed biomechanically more efficient movements than controls, but only when switching movements between hands. This effect correlated with disease severity, such that patients with more severe motor symptoms had a smaller hand path priming effect. We propose that the basal ganglia mediate recycling of movement parameters across subsequent actions.
\end{abstract}

Key words: basal ganglia; motor efficiency; motor planning; Parkinson's disease; priming

\section{Significance Statement}

The human motor system recycles motor parameters of previous actions when programming new actions, promoting efficient motor behavior. Here, we investigated the contribution of the basal ganglia to this transfer of motor parameters over subsequent actions. We assessed motor recycling by analyzing kinematic movement parameters during sequential hand movements that involved either a switch or no switch between hands. Compared with matched controls, Parkinson's patients were impaired in transferring previously used motor parameters to new actions, but only when switching actions between hands. This suggest that the basal ganglia are important for motor recycling, and that the impaired ability of Parkinson's patients to perform this computation may result in motor slowing.

Received November 25, 2019; accepted March 22, 2020; First published April 16, 2020.

M.F., R.P.R.D.v.d.W., R.S., and I.T. declare no competing interests. R.C.H. serves on the clinical advisory board of Cadent Therapeutics and received honoraria from AbbVie. B.R.B currently serves as Associate Editor for the Journal of Parkinson's Disease, serves on the editorial of Practical Neurology and Digital
Biomarkers, has received honoraria from serving on the scientific advisory board for Abbvie, Biogen, UCB, and Walk with Path, and has received fees for speaking at conferences from AbbVie, Zambon, Roche, GE Healthcare, and Bial.

Author contributions: R.P.R.D.v.d.W., B.R.B., I.T., and R.C.H. designed research; R.P.R.D.v.d.W. and R.S. performed research; M.F. and R.P.v.d.W. analyzed data; M.F., R.P.R.D.v.d.W., I.T., and R.C.H. wrote the paper. 


\section{Introduction}

The basal ganglia have an important role in organizing transitions between subsequent actions (Garr, 2019). For example, it has been suggested that the basal ganglia bind sequential motor elements into "chunks" during motor learning (Graybiel, 1998; Wymbs et al., 2012), allowing groups of individual movements to be prepared and executed as a single motor program (Graybiel, 1998; Halford et al., 1998; Wymbs et al., 2012). The basal ganglia are also involved in switching between motor and cognitive demands of a task (Aarts et al., 2010), and, more generally, in switching toward novel behavior (Redgrave and Gurney, 2006). Patients with Parkinson's disease (PD), who have basal ganglia dysfunction (Kish et al., 1988), are behaviorally impaired in action sequencing (Benecke et al., 1987), chunking (Tremblay et al., 2010), and shifting between subsequent actions (Cools et al., 1984; Hayes et al., 1998; Helmich et al., 2009). Recent evidence has highlighted a general principle that might underlie those impairments: previous movements can influence the parameters of subsequent movements over a timescale of seconds. Multiple experiments have shown that re-using motor parameters over consecutive actions may minimize computational demands of motor planning, promoting efficient motor behavior (Jax and Rosenbaum, 2007; van der Wel et al., 2007; Hesse et al., 2008; Dixon and Glover, 2009; Tang et al., 2015). However, it is not known how the motor system re-uses motor parameters of previous actions. Here, we assess the contribution of the basal ganglia in transferring motor parameters over subsequent actions.

The neural architecture of the basal ganglia is well suited to incorporate recent motor history into newly programmed actions. First, the basal ganglia contain multiple recurrent loops between individual nuclei (Taverna et al., 2008; Redgrave et al., 2010; Díaz-Hernández et al., 2018) and between the basal ganglia and the cortex (Alexander et al., 1986). These loops are important for the formation of short-term motor memory (Berns and Sejnowski, 1998), which is necessary for relaying information from previous actions to subsequent actions and thus crucial for the efficient re-use of motor parameters across consecutive actions. Second, the direct and indirect pathways through the basal ganglia allow transitions between subsequent actions by facilitating (new) cortical motor

R.C.H. was funded by the VENI grant from the Netherlands Organization for Scientific Research 91617077. The Center of Expertise for Parkinson \& Movement Disorders of the Radboud university medical center was supported by a Center of Excellence Grant of the Parkinson's Foundation. B.R.B. was supported by the Netherlands Organization for Scientific Research, the Michael J Fox Foundation, UCB, Abbvie, the Stichting Parkinson Fonds, the Hersenstichting Nederland, the Parkinson's Foundation, Verily Life Sciences, Horizon 2020, the Topsector Life Sciences and Health, and the Parkinson Vereniging.

Correspondence should be addressed to Matthias Fritsche at m.fritsche@donders.ru.nl.

https://doi.org/10.1523/ENEURO.0492-19.2020 Copyright @ 2020 Fritsche et al.

This is an open-access article distributed under the terms of the Creative Commons Attribution 4.0 International license, which permits unrestricted use, distribution and reproduction in any medium provided that the original work is properly attributed. representations through the direct pathway, while inhibiting (previous) motor representations through the indirect pathway (Downes et al., 1993; Hayes et al., 1998). This anatomic configuration appears well suited for organizing switches between subsequent actions while re-using elements of previous actions kept in motor memory. Such a process would be critical for recycling motor parameters across different actions. Here, we test the possible role of the basal ganglia in re-using motor parameters shared across subsequent actions. We consider early-stage PD as a model of predominantly basal ganglia dysfunction, testing the prediction that PD patients should be impaired in transferring motor parameters over subsequent actions, especially during action switching.

The study uses a previously validated behavioral task (van der Wel et al., 2007), showing that when participants move their hand over an obstacle, in the context of a sequence of aiming movements, they continue to make unnecessarily large movements even after the obstacle has been cleared ("hand path priming effect"). This effect is present even when participants clear the obstacle with one hand and continue making aiming movements with the other hand. This observation suggests that the hand path priming effect is driven by central motor representations, rather than by biomechanical factors. More generally, this effect suggests that humans minimize changes in motor planning between subsequent movements, sometimes at the expense of biomechanical costs. Importantly, recycling of motor parameters in this task requires previous parameters to be maintained in short-term memory and, in the case of switching actions between hands, to be generalized across different actions. Here, we compare the hand path priming effect between 16 PD patients off dopaminergic medication and 16 matched healthy controls. We expected a reduced hand path priming effect in PD patients compared with healthy controls, particularly when switching actions across hands. The prediction that hand path priming in PD patients should be particularly affected when switching actions between hands derives from the evidence that the basal ganglia play a critical role in action selection and switching (Redgrave et al., 1999; Humphries et al., 2006), in line with frequently observed behavioral impairments of PD patients when shifting between subsequent actions (Cools et al., 1984; Hayes et al., 1998; Helmich et al., 2009).

\section{Materials and Methods}

\section{Participants}

Sixteen patients with PD and 16 healthy controls participated in the study (Table 1). Age and gender did not differ between groups $(p>0.05)$. All participants were right-handed. We recruited controls from the local community and patients through the neurologic outpatient clinic of the Radboud University Medical Center.

Patients were included when they had PD, diagnosed according to the United Kingdom Brain Bank criteria, and asymmetric symptoms mainly on the right side of the body. In this way, we were able to test whether task performance would be different for the most-affected and 
Table 1: Participant characteristics

\begin{tabular}{lll}
\hline & PD patients & Controls \\
Gender (male/female) & $7 / 9$ & $10 / 6$ \\
Age (in years, mean \pm SD) & $60 \pm 6.6$ & $57 \pm 6.5$ \\
UPDRS III (mean \pm SD) & $28.3 \pm 7.4$ & - \\
Hoehn and Yahr & $1(n=1)$ & - \\
& $1.5(n=1)$ & \\
& $2(n=8)$ & \\
& $2.5(n=5)$ & \\
& $3(n=1)$ & - \\
Frontal assessment battery & $17.3 \pm 1.1$ & \\
$\quad$ (FAB, mean \pm SD) & & - \\
Disease duration (in years, & $3.2 \pm 1.5$ & \\
$\quad$ mean \pm SD) & &
\end{tabular}

UPDRS III refers to the motor section of the unified PD rating scale, which has a maximum score of 108 points. The Hoehn and Yahr scale considers five disease stages; stage 2 refers to "bilateral involvement without impairment of balance." the frontal assessment battery has a maximum score of 20 points.

least-affected side. Exclusion criteria were: severe action tremor or dyskinesias (to avoid interference with the task), cognitive dysfunction (i.e., mini-mental state examination $<24$ ), and neurologic comorbidity. Patients were at a relatively early stage of the disease. All patients were tested in a practically defined OFF state, i.e., at least $12 \mathrm{~h}$ after their last dose (Langston et al., 1992). The Central Committee on Research involving Human Subjects approved the experimental procedure. All participants gave written informed consent before the start of the study. The current study was not preregistered.

\section{Apparatus and procedure}

Figure 1 shows the experimental setup, which replicated the experiment by van der Wel et al. (2007). Participants sat at a table with a board on which six targets were evenly spaced in a semicircle. Including six targets assured that participants completed multiple movements on each side of the midline, and one movement across the midline, while making sizeable arm movements within reachable space. Participants held a wooden dowel in either or both of their hands, depending on the experimental condition. The experimenter instructed participants that they were to transport this dowel from target to target using a "jumping" movement. In the experimental conditions, an obstacle stood between either the leftmost or rightmost target pair. Participants were to clear the obstacle by moving over it with the dowel. They were instructed not to move around the obstacle. No obstacle was present in the control conditions. We externally paced the movement rhythm using an auditory metronome set to $1 \mathrm{~Hz}$ to avoid differences in movement frequencies across participants and groups, which may influence the magnitude of the hand path priming effect (Jax and Rosenbaum, 2009). We recorded participants' movements in three dimensions ( $x-, y$-, and $z$-axes) with a Polhemus Liberty at a sampling rate of $200 \mathrm{~Hz}$. A sensor was positioned between thumb and index finger of each hand, respectively. Before the experiments started, participants completed a practice block, lasting a few minutes, in which they moved in time with the metronome while no obstacle was present. The experiment lasted $\sim 32 \mathrm{~min}$.
We manipulated obstacle presence (absent or present) and whether participants performed the movements with one or both hands (unimanual or bimanual). In each block, participants were asked to start the movement either on the leftmost or rightmost target. If they started on the left, in the unimanual conditions participants moved with their left hand from target to target in the rightward direction, all the way to the farthest target on the right, and then back to the left, tapping all the targets in between, to then return rightward again. When starting on the right, participants used their right hand and the sequence was reversed. For the bimanual conditions, participants held a dowel in each hand. They performed the first movement with the hand corresponding to side of the starting location. In the obstaclepresent condition, this entailed moving that hand over the obstacle. They then continued the sequence with their other hand, which rested on the target next to the target pair between which the obstacle stood. Once they returned to that target, they moved over the obstacle with their initial hand again. In obstacle-present blocks, obstacles were always placed next to the starting location. Participants were asked to move back and forth five times to collect meaningful averages for each movement and to reduce the influence of start-up effects. Figure 1 shows example trajectories in three-dimensional space for obstacle-absent and obstaclepresent blocks in the unimanual condition (Fig. 1A) and example trajectories for a control and a patient in each of the four experimental conditions (Fig. 1B).

\section{Experimental design}

The experiment implemented a $2 \times 2 \times 2$ full factorial design with factors transfer (unimanual vs bimanual), obstacle (absent vs present), and laterality (block started with left vs right hand). Each of the eight conditions was tested in two blocks, resulting in a total of 16 blocks of $\sim 2$ min each. The order of conditions was pseudo-randomized across the experiment: the obstacle-present versus obstacle-absent conditions always alternated, and half of the participants started with the obstacle-absent condition. Each block contained at least five back-and-forth sequences consisting of 10 movements each. The hand path priming effect was quantified as the average difference in peak movement height for the obstacle-present minus the obstacle-absent conditions. This effect was calculated over 10 repetitions (two blocks $\times$ five movement sequences), separately for each of the conditions. We only used the first three movements after clearing the obstacle in our analyses. This was done for two reasons. First, by doing so, we used only movements participants made after they cleared the obstacle but before they changed their movement direction (due to reaching the last target in a given direction). Second, the results by van der Wel et al. (2007) indicated that differences in peak heights largely leveled off after the first three postobstacle movements. Including additional movements in the analysis could thus reduce the sensitivity to the hand path priming effect.

\section{Statistical analysis}

Hand path priming

We statistically compared the hand path priming effect in a $2 \times 2 \times 2 \times 3$ ANOVA with within-subject factors 
A

\section{Unimanual condition}

No obstacle

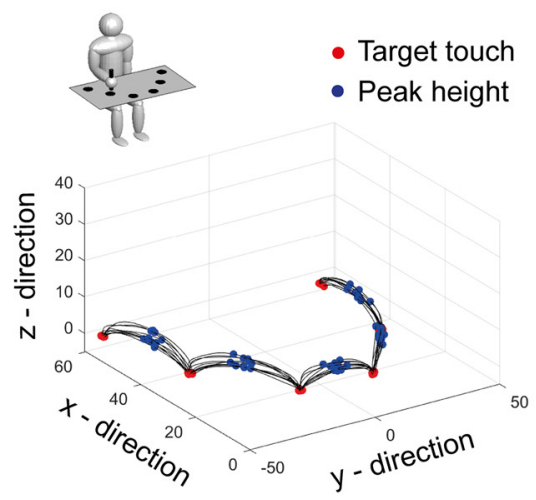

B
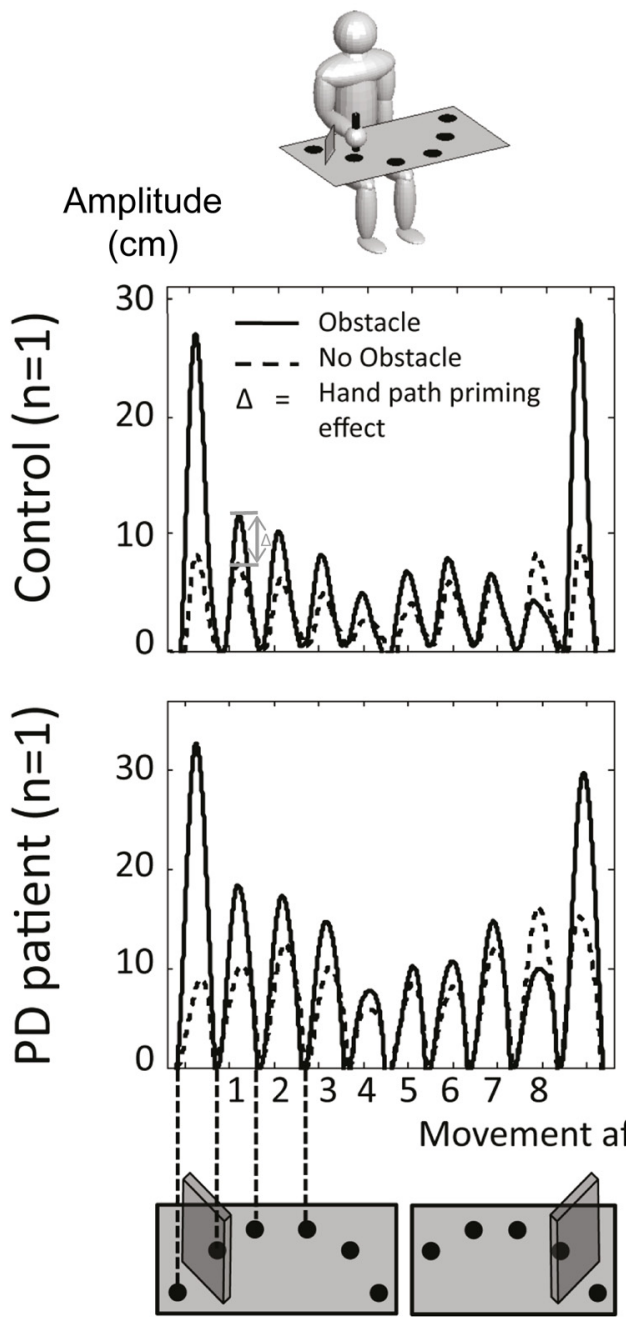

Obstacle

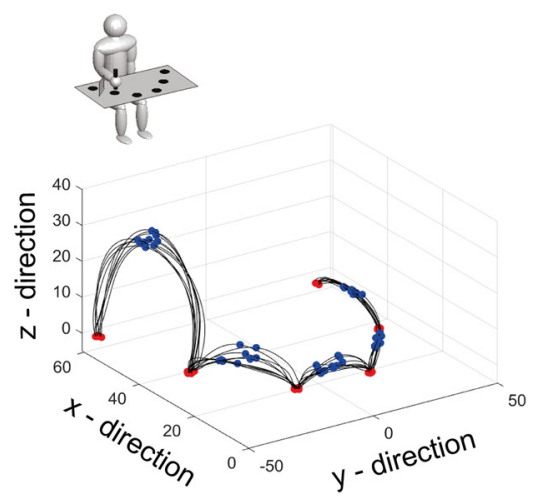

Bimanual
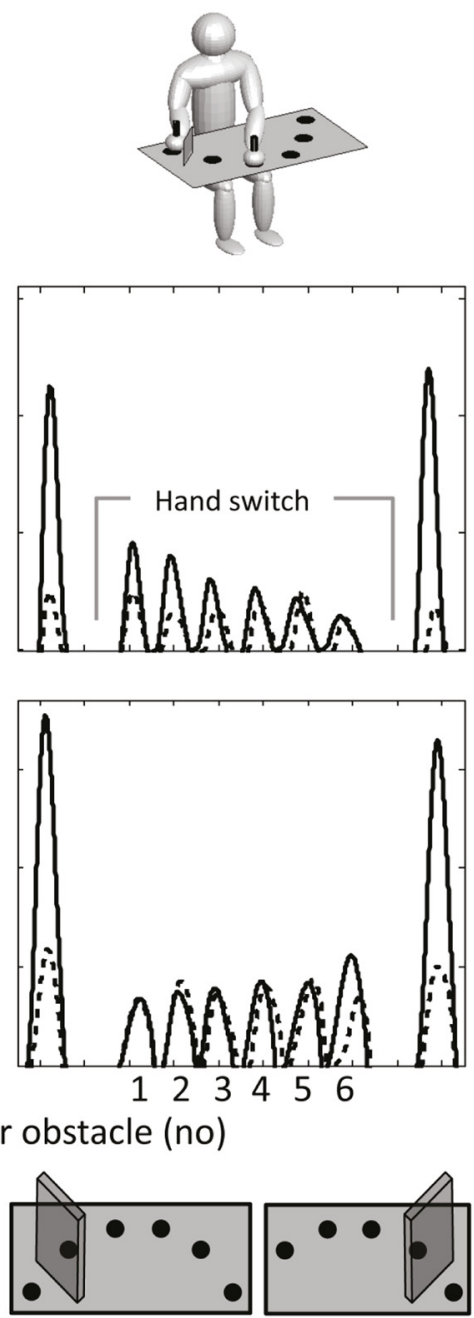

Figure 1. Overview of the experimental setup and example trajectories. $\boldsymbol{A}$, Example trajectories of one control participant in threedimensional space for obstacle-absent (left panel) and obstacle-present blocks (right panel) in the unimanual condition. Participants performed jumping movements between targets (red dots). The points of peak height between the targets are marked with blue dots. Participants performed five back and forth movements in each block, as indicated by 10 connecting lines between each 
continued

target. Movements were recorded with a sensor positioned between thumb and index finger of each hand, respectively. $\boldsymbol{B}$, Example trajectories for each condition, for one control participant and one PD patient, respectively. The left column shows the unimanual condition, and the right column the bimanual condition. The top row shows the experimental setup. The middle row shows the movement trajectory (averaged across all 10 repetitions) for one control participant and the lower row shows the movement trajectory for one PD patient. The movement trajectories are shown for the "no obstacle" condition (dashed lines), and for the "obstacle" condition (solid lines). The difference between these two conditions is the hand path priming effect. The empty spaces in the bimanual condition (plots on the right side) mark the hand switch. Displayed trajectories correspond to one back and forth sequence along all the targets. This plot shows that in the bimanual condition, the example control participant has a hand path priming effect for the first movement after obstacle clearance (i.e., a difference between the two lines depicting the obstacle present and obstacle absent conditions), while the example PD patient does not have a hand path priming effect.

transfer (unimanual vs bimanual), laterality (block started with left hand vs right hand), and movement (first, second or third movement after obstacle clearance), and between-subjects factor group (PD patients vs controls). We applied a Greenhouse-Geisser correction to correct the degrees of freedom whenever a violation of sphericity was indicated.

Since the magnitude of the hand path priming effect has been shown to depend on the movement amplitude of the initial obstacle-clearing movement (van der Wel et al., 2007), and PD patients and controls may exhibit different amplitudes for this initial movement, we repeated the above analysis, but normalized each participant's hand path priming effect by the individual difference in the initial movement's amplitude between obstacle and no-obstacle conditions.

\section{Hand path priming and disease severity}

We hypothesized that the magnitude of the hand path priming effect in PD patients might be influenced by the patients' disease severity. In order to assess this relationship, we correlated disease severity, quantified as the UPDRS III score, with the hand path priming effect, separately for the first three movement after obstacle clearance in the unimanual and bimanual conditions. Since UPDRS scores are not interval scales, we computed Spearman's rank-order correlations. Furthermore, to control for a potential influence of the movement amplitude of the initial obstacle-clearing movement, we also performed the correlation analysis on the normalized hand path priming effect.

\section{Hand rotations}

Next to the hand path priming effect in movement height, we also studied the effect of obstacle clearance on subsequent hand rotations (pitch and roll). To this end, we calculated the angular differences in hand orientation along the sensor's pitch and roll axis at the movement peaks of the first three movements after obstacle clearance. Analogous to the analysis of the hand path priming effect, we subjected these data to $2 \times 2 \times 2 \times 3$ ANOVAs with within-subject factors transfer (unimanual vs bimanual), laterality (block started with left vs right hand) and movement (first, second or third postobstacle movement), and between-subjects factor group (PD patients vs controls). The purpose of this analysis was to rule out that any effects in hand path priming could be caused by systematic differences in hand rotations, which themselves may affect the height of the sensor attached to the hand.

\section{Movement and dwell times}

To further characterize participants' behavior on the task, we additionally analyzed movement and dwell times (Fig. 2). Movement times were defined as the elapsed time between the moments when participants lifted the dowel from successive targets. For each participant, we averaged movement times across all movements within a complete back and forth movement sequence (10 movements in unimanual condition, eight movements in bimanual condition) and across all sequence repetitions in a given condition (five repetitions per block, with two blocks each), resulting in an average movement time across 100 movements in the unimanual and 80 movements in the bimanual condition, respectively. We statistically compared movement times across conditions in a $2 \times 2 \times 2 \times 2$ repeated-measures ANOVA, with within-subject factors obstacle (obstacle-present vs absent), transfer (unimanual vs bimanual), laterality (block started with left hand vs right hand), and between-subject factor group (PD patients vs controls).

Moreover, we sought to relate dwell times during which participants planned their subsequent movement to the

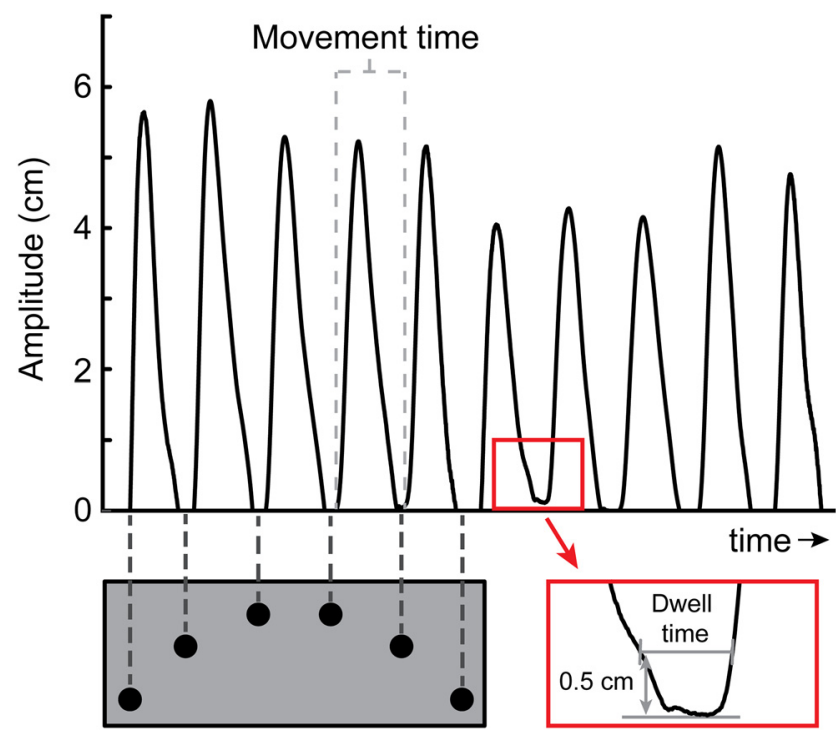

Figure 2. Measurement of movement and dwell times. Movement times were defined as the elapsed time between the moments when participants lifted the dowel from successive targets (gray dotted lines). Dwell times were defined as the time interval during which the dowel was within a $0.5-\mathrm{cm}$ distance above the target (red box). 

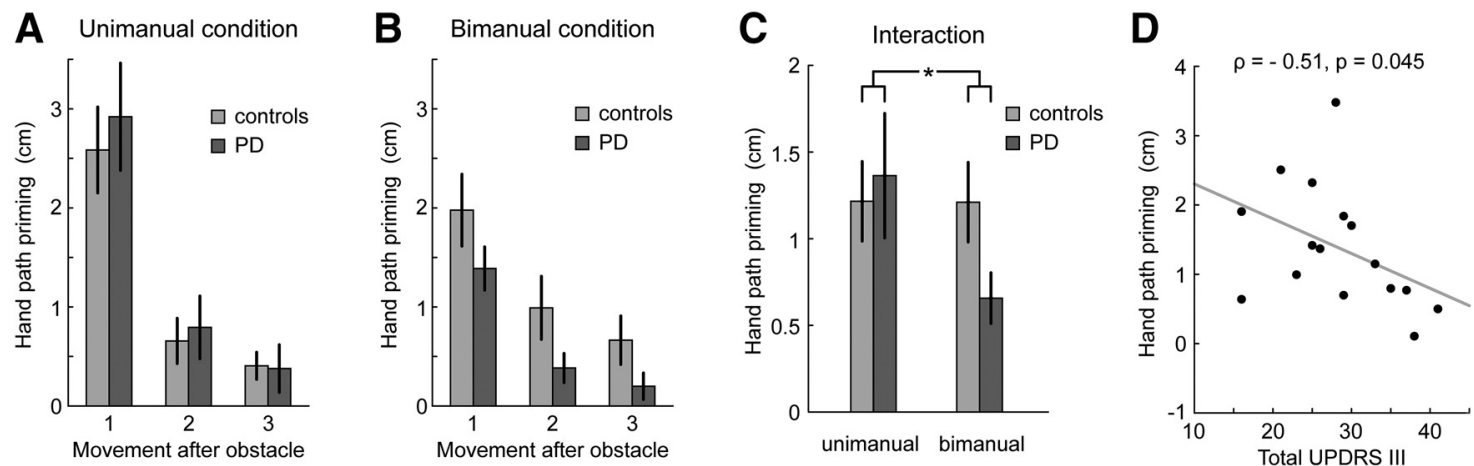

Figure 3. Hand path priming effect. The average difference in peak movement height between the obstacle-present and obstacleabsent conditions is plotted as a function of the movement number after clearing the obstacle, separately for the unimanual $(\boldsymbol{A})$ and bimanual condition $(\boldsymbol{B})$. C . Significant group $\times$ transfer interaction, indicating that PD patients have a reduced hand path priming effect in the bimanual condition, but not in the unimanual condition. $\boldsymbol{D}$, Relationship between disease severity and hand path priming effect. The hand path priming effect for the first postobstacle movement in the bimanual condition decreases with increasing disease severity. Error bars depict SEMs.

hand path priming effect in this movement. Dwell times, i.e., the time that participants rested on each target, were defined as the time interval during which the dowel was within a $0.5-\mathrm{cm}$ distance above the target, where distance is computed along the $z$-axis (i.e., height). We computed dwell times for each of the first three postobstacle targets. We then statistically analyzed the dwell time estimates in a similar ANOVA as described above. Furthermore, to relate movement preparation (dwell time) to movement plan reuse (hand path priming), we calculated correlations between the hand path priming effect and dwell times variables for the first target landing and movement after clearing the obstacle.

\section{Results}

\section{Hand path priming}

Participants (i.e., both patients and controls) showed a pronounced hand path priming effect after clearing the obstacle $\left(F_{(1,30)}=43.749, p<0.001\right.$; Fig. 3$)$. This effect gradually disappeared as participants moved further away from the obstacle (main effect of movement, $F_{(1.175,35.240)}=$ $79.834, p<0.001)$. The hand path priming effect was smaller when participants switched the moving hand after clearing the obstacle (main effect of transfer, $F_{(1,30)}=4.683$, $p=0.039$ ), but it decayed more slowly (interaction between transfer and movement, $\left.F_{(1.379,41.359)}=23.958, p<0.001\right)$. Crucially, the hand path priming effect was smaller in $\mathrm{PD}$ patients than in controls, but only when patients switched between hands after clearing the obstacle (interaction between group and transfer, $\left.F_{(1,30)}=4.551, p=0.041\right)$. In particular, only for bimanual trials, but not for unimanual trials, there was a trend toward smaller priming in PD patients, compared with controls, which, however, did not survive the statistical threshold (bimanual: $F_{(1,30)}=3.122, \quad p=0.087$; unimanual: $\left.F_{(1,30)}=0.121, p=0.731\right)$. In fact, for unimanual trials, a post hoc Bayesian $t$ test revealed moderate evidence for the null hypothesis of no difference between PD patients and versus the hypothesis of a larger hand path priming effect in healthy participants $\left(\mathrm{BF}_{0+}=3.72\right)$. There were no significant differences between movements with the left or the right hand. Together, these analyses demonstrate a reduced hand path priming effect in $\mathrm{PD}$ patients relative to controls, specific to the bimanual condition. Conversely, PD patients and controls exhibit a similar hand path priming effect in the unimanual condition.

Importantly, for the initial obstacle clearing movement, control participants showed a larger movement height difference ( $\Delta$ clearance) between obstacle-present and obstacle-absent trials compared with PD patients (main effect of group, $\left.F_{(1,30)}=8.30, p=0.007\right)$. Furthermore, while control participants exhibited larger $\Delta$ clearance for movements with their dominant right compared with left hand $\left(F_{(1,15)}=9.36, p=0.008\right)$, PD patients had a slight, but not significant, tendency toward smaller $\Delta$ clearance for right compared with left hand movements $\left(F_{(1,15)}=\right.$ $2.42, p=0.14)$, leading to a significant group $\times$ laterality interaction $\left(F_{(1,30)}=11.76, p=0.002\right)$. This likely reflects bradykinesia in the mainly right-side affected PD patients. Crucially, the difference in $\Delta$ clearance between groups was similar for the unimanual and bimanual conditions (no interaction between group and transfer, $F_{(1,30)}=0.87$, $p=0.359$ ) and thus is unlikely to explain the group $x$ transfer interaction in the hand path priming effect. Nevertheless, we conducted a control analysis to rule out that differences in hand path priming were driven by systematic differences in the initial obstacle clearing movement. Specifically, we expressed the hand path priming effect relative to the height difference in the initial movements of obstacle-present and absent trials. Crucially, in agreement with the above results, the analysis of this relative hand path priming effect again showed a significant group $\times$ transfer interaction $\left(F_{(1,30)}=6.97, p=0.013\right)$.

\section{Hand path priming and disease severity}

We correlated the hand path priming effect for the first movement after the obstacle with disease severity (UPDRS motor score). There was a significant negative correlation for the bimanual condition (Spearman's $\rho=$ $-0.50, p=0.045$; Fig. $3 D$ ), but not the unimanual condition. This correlation was similarly present, after 

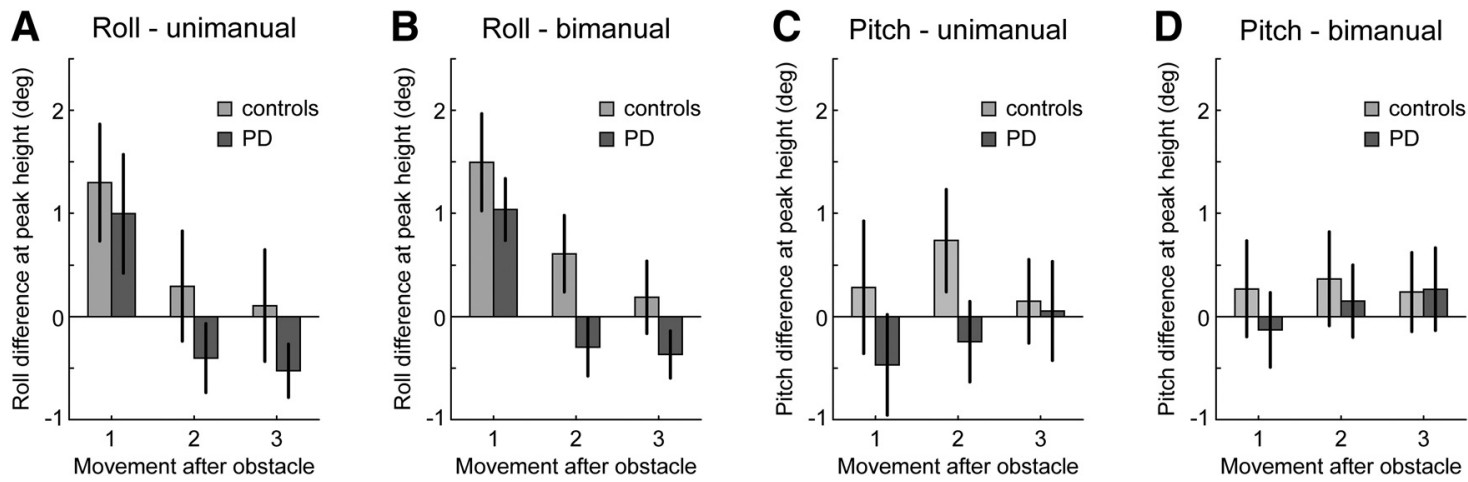

Figure 4. Hand rotations. Hand rotation differences between obstacle-present and absent trials at the highest points of the first three movements after clearing the obstacle. There are systematic roll rotation differences between obstacle-present and absent trials, both in the unimanual $(\boldsymbol{A})$ and bimanual conditions $(\boldsymbol{B})$. These differences decrease as participants move further away from the obstacle. No such patterns are found for pitch rotation differences $(\boldsymbol{C}, \boldsymbol{D})$. There are no significant differences in hand rotations between PD patients and controls, and overall rotation differences are too small to exert a noticeable effect on the height estimate of the sensor. Error bars depict SEMs.

controlling for individual movement height differences of the initial obstacle-clearing movement ( $\Delta$ clearance) between obstacle-present and obstacle-absent trials (Spearman's $\rho=-0.50, p=0.049$ ). Thus, the greater a patient's disease severity, the smaller his or her hand path priming effect was. There were no significant correlations between disease severity and the other movements after clearing the obstacle (ps $>0.3$ ).

\section{Hand rotations}

For the analysis of hand rotations, we excluded two participants of the control group, who showed exceptionally large differences in hand rotations between the obstaclepresent and absent conditions $\left(>60^{\circ}\right.$ roll or pitch difference in at least one condition). Importantly, after excluding these two participants, the group $\times$ transfer interaction in the hand path priming effect remained significant $\left(F_{(1,28)}=6.72\right.$, $p=0.015$ ).

Overall, rotation differences between the obstacle-present and absent trials were minute $\left(<1.5^{\circ}\right.$ on average; Fig. 4 ), and as such they were unable to account for any appreciable differences in sensor heights across conditions. Furthermore, there were no significant differences in hand rotations across groups. Therefore, it is unlikely that differences in hand path priming reported above were due to systematic differences in hand rotations, rather than a priming effect in movement amplitudes. However, although hand rotation differences were small, we found a main effect of movement on roll rotation differences $\left(F_{(1.18,33.12)}=\right.$ $34.31, p<0.001)$, with roll rotation differences gradually decreasing as participants moved further away from the obstacle. This mirrored the decreasing hand path priming effects in movement amplitudes with increasing distance from the obstacle, hinting that the recycling of movement parameters might not be limited to movement amplitudes, but may include hand rotations. Consequently, in a followup analysis, we correlated the hand rotation differences between obstacle-present and absent trials with the hand path priming effect in movement amplitudes across participants. We found strong correlations for roll rotations, both in the unimanual and bimanual condition (unimanual: $r=0.66, p=7.14 \mathrm{e}-5$; bimanual: $r=0.82, p=1.72 \mathrm{e}-8)$. Correlations were not different between PD patients and controls. There were no significant effects involving pitch rotations. Overall, this suggests that while hand rotation differences were too small to bias movement amplitude estimates in any appreciable way, in the current experiment the recycling of movement parameters was likely not limited to movement amplitudes, but extended to roll rotations of the hand. However, in contrast to priming of movement amplitudes, the effects in roll rotation were not noticeably different between PD patients and controls.

\section{Movement and dwell times}

Participants closely followed the metronome period of $1 \mathrm{~Hz}\left(\mathrm{M}_{\text {Controls }}=976 \mathrm{~ms} ; \mathrm{M}_{\text {Patients }}=985 \mathrm{~ms}\right)$. They exhibited shorter movement times in the bimanual compared with the unimanual condition (main effect of transfer: $F_{(1,30)}=$ $32.668, p<0.001)$ and this was especially pronounced for controls (transfer $\times$ group interaction $\left(F_{(1,30)}=6.12, p=\right.$ $0.019)$. Within the bimanual condition, there was a trend toward longer movement times for PD patients compared with controls, which did not survive the statistical threshold (main effect of group, $F_{(1,30)}=3.75, p=0.062$ ). Importantly, there was no significant correlation between the patients' movement times and their hand path priming effect for the first postobstacle movement $(r=-0.39, p=0.14)$, suggesting that the patients' increased movement times and decreased hand path priming effects in the bimanual condition were unrelated. Furthermore, when computing movement times only for the first three postobstacle movements, for which the hand path priming effect was computed, both groups showed very similar movement times in both unimanual $\left(\mathrm{M}_{\text {Controls }}=968 \mathrm{~ms}\right.$; $M_{\text {Patients }}=967 \mathrm{~ms}$; $\left.F_{(1,30)}=0.042, p=0.84\right)$ and bimanual conditions ( $\left(\mathrm{M}_{\text {Controls }}=\right.$ $\left.1017 \mathrm{~ms} ; M_{\text {Patients }}=1005 \mathrm{~ms} ; F_{(1,30)}=1.466, p=0.23\right)$. For detailed description of movement times, see Tables 2, 3 .

In addition to the analysis of movement times, we also analyzed dwell times on the targets between movements. Dwell times were defined as the time interval during which 
Table 2: Movement times (in milliseconds), computed as the elapsed time between the moments when participants lifted the dowel from successive targets

\begin{tabular}{lllll}
\hline & & & & Bimanual \\
& Obstacle-absent & Unimanual & Obstacle-absent & Obstacle-present \\
Controls & $996(2)$ & $987(2)$ & $960(2)$ & $960(4)$ \\
Patients & $993(3)$ & $990(6)$ & $973(4)$ & $985(17)$
\end{tabular}

For each participant, we averaged movement times across all movements in a complete back and forth movement sequence (10 movements in unimanual condition, eight movements in bimanual condition) and across all sequence repetitions in a given condition (five repetitions per block, with two blocks each). Movement times in this table present averages over the factor laterality (left/right hand). Numbers in parenthesis indicate SEM.

the dowel was within a $0.5-\mathrm{cm}$ distance above the target. Similar to the hand path priming analysis, we only analyzed dwell times of the first three movement after clearing the obstacle. We found that PD patients dwelled significantly shorter on the target location compared with controls (main effect of group: $F_{(1,30)}=4.47, p=0.043$ ). This is likely a result of compensating for slower movements in between targets due to bradykinesia. Furthermore, we found a significant obstacle $\times$ movement interaction $\left(F_{(1.3 .39 .00)}=70.01, p<0.001\right)$, which indicated that, specifically in the conditions in which an obstacle was present, dwell times increased as participants moved further away from the obstacle. We also observed main effects of obstacle $\left(F_{(1,30)}=95.09, p<0.001\right)$ and of movement $\left(F_{(1.22,36.56)}=27,93, p<0.001\right)$. Finally, there was a significant transfer $\times$ movement interaction $\left(F_{(1.21,36.33)}=6.29\right.$, $p=0.012$ ), indicating that the increase in dwell times with distance from the obstacle was particularly pronounced in the unimanual condition. Importantly, there was no significant interaction involving group, suggesting that apart from generally shorter dwell times, PD patients' dwell times followed the same patterns as those of controls. For detailed description of dwell times, see Table 4.

To relate movement preparation to movement plan reuse, we calculated partial correlations between the hand path priming effect and dwell time variables for the first target landing and movement after clearing the obstacle, while controlling for differences in movement heights of the initial obstacle clearing movement between obstacle-present and absent trials ( $\Delta$ clearance), which may both affect dwell times and the hand path priming effect. We found that in the unimanual condition the hand path priming effect was negatively correlated to dwell time ( $r=$ $-0.48, p=0.007$ ), indicating that shorter movement preparation was associated with a stronger priming effect. In the bimanual condition this correlation was not significant over all participants $(r=-0.19, p=0.30)$, but was only significant for the PD patient group $(r=-0.58, p=0.024)$. See Table 5 for a summary of all correlations. Together, these results hint that the re-use of motor parameters from a previous action (hand path priming effect) may decrease the time to program the next action (dwell time).

\section{Discussion}

We investigated how relatively early stage PD patients, whose pathophysiology was presumably confined to a predominant basal ganglia dysfunction, incorporate an element of a previous action (i.e., movement amplitude) into a subsequent action. To this end, we used a previously validated behavioral task (van der Wel et al., 2007), showing that when participants move their hand over an obstacle, in the context of a sequence of aiming movements, they continue to make unnecessarily large movements even after the obstacle has been cleared (hand path priming effect). Compared with healthy controls, PD patients had a reduced hand path priming effect, but only when they switched between hands. Furthermore, the magnitude of the bimanual hand path priming effect decreased with greater disease severity. This finding suggests that PD patients are impaired in adjusting previously used motor parameters to new actions, extending previous studies that regarded action switching as a transition between two discrete motor programs (Cools et al., 1984; Helmich et al., 2009). This suggests that fronto-striatal recycling of movement parameters contributes to efficient motor control. We speculate that the motor slowing characteristic of PD might result at least in part from this impaired motor recycling process.

\section{The hand path priming effect in PD}

Both healthy controls and PD patients showed a clear hand path priming effect, indicating that they continued to make larger movements than necessary after clearing an obstacle with the same or the other hand. Participants recycled a kinematic element of the previous action, movement amplitude, when programming the next. The fact that the hand path priming effect was also present when switching between hands rules out that the priming effect is exclusively caused by mechanical factors, such as muscle relaxation (van der Wel et al., 2007). Instead, the hand path priming effect likely reflects a central property of movement planning, pertaining to movement features that generalize across different effectors and spatial

Table 3: Movement times (in milliseconds) averaged over the first three postobstacle movements

$\begin{array}{lllll} & & & & \text { Bimanual } \\ & \text { Obstacle-absent } & \text { Unimanual } & \text { Obstacle-absent } & \text { Obstacle-present } \\ \text { Controls } & 983(7) & 953(8) & 1036(8) & 998(8) \\ \text { Patients } & 996(3) & 938(8) & 1019(9) & 990(6)\end{array}$

Movement times were defined as the elapsed time between the moments when participants lifted the dowel from successive targets. Numbers in parenthesis indicate SEM. 
Table 4: Dwell times (in milliseconds) computed for the first three postobstacle movements

\begin{tabular}{|c|c|c|c|c|c|c|c|c|c|c|c|c|}
\hline \multirow{2}{*}{ Movement } & \multicolumn{6}{|c|}{ Unimanual } & \multicolumn{6}{|c|}{ Bimanual } \\
\hline & \multicolumn{3}{|c|}{ Obstacle-absent } & \multicolumn{3}{|c|}{ Obstacle-present } & \multicolumn{3}{|c|}{ Obstacle-absent } & \multicolumn{3}{|c|}{ Obstacle-present } \\
\hline Controls & $364(31)$ & $378(30)$ & $390(33)$ & $235(27)$ & $327(30)$ & $365(34)$ & $339(24)$ & 397 (29) & $350(28)$ & $218(26)$ & $318(25)$ & $313(28)$ \\
\hline
\end{tabular}

Dwell times were defined as the time interval during which the dowel was within a 0.5 -cm distance above the target. Numbers in parenthesis indicate SEM

locations. This inference is supported by recent data showing that different movement parameters (i.e., spatial and temporal features of movement sequences) are independently encoded in the motor system, and can be flexibly transferred from trained to novel sequences (Kornysheva and Diedrichsen, 2014). Planning of upcoming movements is more efficient by changing just those features that distinguish upcoming movements from recent movements, rather than starting "from scratch" each time a movement is required (Jax and Rosenbaum, 2007; Rosenbaum et al., 2007). Our design was optimized to test for the transfer of movement amplitude over sequential actions, while we imposed a fixed rhythm and fixed targets. However, it is likely that the recycling of movement parameters is not limited to movement amplitude in general. For instance, we found that roll rotations of the hand systematically differed between obstacle-present and absent trials for postobstacle movements, even when participants switched hands. Furthermore, this effect in roll rotations correlated with the hand path priming effect in movement amplitudes, suggesting a similar carryover for both motor parameters. However, whether the carryover in roll rotation is a mere consequence of larger movements due to amplitude priming, or whether movement parameters such as hand rotations can be independently primed remains a question for future research.

The current study controlled for several potential confounds related to comparing PD patients with controls. First, the main outcome measure (hand path priming effect) is the difference in movement amplitude between obstacle-present and absent conditions (van der Wel et al., 2007), controlling for systematic alterations in body posture between groups. Second, the differential hand path priming effect in PD is not a trivial consequence of the smaller movements performed by the PD patients (Desmurget et al., 2003). The between-groups difference in hand path priming effect was specific to the bimanual condition, and normalizing the hand path priming effect to

Table 5: Partial correlations between dwell times and the hand path priming effect (first postobstacle movement), accounting for differences in movement heights of the initial obstacle clearing movement between obstacle-present and absent conditions ( $\Delta$ clearance)

\begin{tabular}{lll}
\hline & Unimanual & Bimanual \\
All participants & $\rho=-0.48$ & $\rho=-0.19$ \\
Controls & $p=0.007$ & $p=0.30$ \\
Patients & $\rho=-0.45$ & $\rho=-0.01$ \\
& $p=0.09$ & $p=0.97$ \\
& $\rho=-0.53$ & $\rho=-0.58$ \\
& $p=0.04$ & $p=0.02$
\end{tabular}

the initial movement, which primed subsequent movements, did not change that finding. Third, movement frequency was controlled by using a metronome to time the movements to an equal rhythm in both groups. This is important, because previous work has shown that the hand path priming effect decreases with increasing intervals between subsequent actions (Jax and Rosenbaum, 2009). However, it should be noted that although both groups were generally well able to follow the imposed rhythm, there was a trend toward longer movements in the bimanual condition for PD patients compared with controls. However, this difference was very small $(19 \mathrm{~ms})$ and the patients' movement times did not correlate with their hand path priming effect. Furthermore, when computing movement times only for the first three postobstacle movements, for which the hand path priming effect was quantified, both groups showed very comparable movement times. This suggests that longer movement times cannot account for the reduced hand path priming effect of PD patients in the bimanual condition. Furthermore, the similar movement and dwell times for PD patients and controls indicate that PD patients did not strongly suffer from traditional switch costs, typically characterized by increased response times following action switches. Therefore, it seems unlikely that the reduced hand path priming effect, measured in movement amplitudes, can be explained by general difficulties of switching movements between hands. Rather, the current results point toward a selective impairment in transferring motor parameters across subsequent different motor actions, which constitutes a novel type of switch cost, extending our current knowledge of switch costs in PD.

\section{The role of the fronto-striatal circuit in movement transitions}

The impaired ability of PD patients to recycle action parameters for subsequent actions was only apparent for action switches, i.e., when participants cleared the obstacle with one hand and continued with the other. This fits with extensive literature showing that the basal ganglia are involved in switching between movements (Cools et al., 1984; Hayes et al., 1998; Helmich et al., 2009; Garr, 2019). The reduced ability to recycle motor elements from previous actions, as shown here, may force PD patients to plan new actions from scratch, causing behavioral delays (switch costs). The observed inverse relationship between the hand path priming effect (recycling) and dwell times (switch costs), when computed over all participants in the unimanual condition, is consistent with this notion. In line with the idea of impaired motor recycling in PD patients, behavioral studies have shown that PD patients re- 
program reaching movements during its execution (Gentilucci and Negrotti, 1999), suggesting impaired motor working memory that causes motor programs to decay during their time course. Crucially, this effect depended on the context in which the movement occurred: PD patients were perfectly able to adapt ongoing reaching movements, but they were severely impaired when adapting their movement trajectory by switching toward a new movement (Desmurget et al., 2004).

Several mechanisms may be responsible for the impaired ability of PD patients to organize transitions between consecutive actions. A first mechanism may involve the impairment of motor working memory, which is thought to rely on the presence of recurrent loops (Berns and Sejnowski, 1998). Indeed, there are multiple recurrent loops in the basal ganglia circuit, for example the short-range loop between the external globus pallidus (GPe) and subthalamic nucleus (STN; Redgrave et al., 2010), the long-range loop between the basal ganglia and the frontal cortex (Alexander et al., 1986; Redgrave et al., 2010), and recurrent connections between the thalamus and the striatum (Díaz-Hernández et al., 2018). This allows the basal ganglia to support "competitive cueing": holding a second movement plan in abeyance while the first movement is being executed (Bhutani et al., 2013). In experimental parkinsonism, it has been shown that recurrent connections in the basal ganglia circuit are disrupted (Taverna et al., 2008) and sequential activity in the basal ganglia is abolished (Jáidar et al., 2010). Furthermore, PD patients have impaired activity in several nodes along the fronto-striatal loop, such as the supplementary motor area (SMA; Wu et al., 2010), which has a specific role in supporting bimanual sequences (Johnson et al., 1998). Thus, the loss of recurrent connections in the fronto-striatal circuit of PD patients may impair motor working memory, resulting in a rapid decay of motor parameters from previous actions. A second mechanism may involve the increased suppression of previous movements in PD. More specifically, striatal dopamine depletion in PD reduces processing in the direct pathway, which facilitates selection of new movements, and it increases processing in the indirect pathway, which inhibits previous movements (Downes et al., 1993; Hayes et al., 1998). The increased suppression of previous actions through the indirect pathway may thus prevent recycling of previous motor elements (Baladron et al., 2019). Taken together, both impaired motor memory and increased suppression of previous actions by the indirect pathway may explain the impaired recycling of previous motor elements in PD observed here.

\section{Inefficient motor planning in PD}

Our findings indicate that PD patients were less inclined than controls to perform movements of greater amplitude than strictly necessary, although this may minimize planning costs (Jax and Rosenbaum, 2009). This fits with a large body of evidence showing that basal ganglia dysfunction in PD leads to a default amplitude setting that is lower than what is needed (Beckley et al., 1993; Horak et al., 1996; Desmurget et al., 2003). It has also been suggested that the basal ganglia estimate the "cost-togo" during the execution of a motor task, balancing the value and costs of motor commands (Shadmehr and Krakauer, 2008). This idea is based on a study where PD patients and controls were asked to make accurate reaching movements of specified speeds (Mazzoni et al., 2007). While PD patients had normal spatial accuracy in each condition, they required more trials than controls to accumulate the required number of movements in each speed range. This was interpreted as a "reluctance" to execute movements requiring greater effort, despite preserved spatial accuracy. Our findings suggest that basal ganglia dysfunction in PD may lead to wrong priorities (Bloem et al., 2006), i.e., reduction of biomechanical costs at the expense of inefficient motor planning.

\section{Limitations and future research}

Our study has several limitations. First, it might be argued that PD is not an adequate model of basal ganglia dysfunction, since other cerebral systems are also impaired in these patients. By including relatively earlystage PD patients (average disease duration 3.2 years) without cognitive dysfunction, we attempted to reduce the influence of such impairments, such as cortical Lewy body pathology occurring in more advanced PD (Braak et al., 2003). Surprisingly, the hand path priming effect was similar for both hands, although all patients had more motor symptoms on their right side than on their left side. This may be caused by the fact that, despite an asymmetry, 14 out of 16 PD patients had bilateral motor symptoms. Furthermore, even the clinically unaffected side was likely also influenced by the underlying disease process, as indicated by quantitative bradykinesia tests performed in limbs of PD patients that were deemed unaffected based on clinical assessments (Haaxma et al., 2010). Since there is $\sim 50-90 \%$ nigro-striatal cell loss at the onset of clinical symptoms (Kordower et al., 2013), this means that almost all patients already had substantial and bilateral basal ganglia dysfunction. Future studies may use neuroimaging in healthy participants, or apply focused basal ganglia lesions in primates, to further test the role of the basal ganglia in motor recycling. Second, in our design an action switch was always a switch between two different hands. Future studies may test whether the motor recycling impairment in PD is specific to a transition between effectors, or is also present when switching between different actions within one effector. Third, while the current study provides evidence for systematic differences in the recycling of motor parameters between PD patients and controls, the effect size of these differences seems to be relatively small. Since the current conclusions are based on a relatively small sample size, we deem it important that future studies will replicate and extend the current findings in larger sample sizes. This would be particularly helpful for providing a more precise estimate of the true effect size. Nevertheless, the current study, involving carefully screened PD patients, may provide a valuable starting point for understanding the role of the basal ganglia in motor recycling and the accompanied deficits in PD patients. 


\section{Conclusion}

Parkinson's patients were impaired in recycling motor parameters shared across subsequent actions, specifically, in the context of action switching. We suggest that the basal ganglia are important for motor recycling, and that the impaired ability of Parkinson's patients to perform this computation may result in inefficient motor behavior.

\section{References}

Aarts E, Roelofs A, Franke B, Rijpkema M, Fernández G, Helmich $R C$, Cools R (2010) Striatal dopamine mediates the interface between motivational and cognitive control in humans: evidence from genetic imaging. Neuropsychopharmacology 35:1943-1951.

Alexander GE, DeLong MR, Strick PL (1986) Parallel organization of functionally segregated circuits linking basal ganglia and cortex. Annu Rev Neurosci 9:357-381.

Baladron J, Nambu A, Hamker FH (2019) The subthalamic nucleusexternal globus pallidus loop biases exploratory decisions towards known alternatives: a neuro-computational study. Eur J Neurosci 49:754-767

Beckley DJ, Bloem BR, Remler MP (1993) Impaired scaling of long latency postural reflexes in patients with Parkinson's disease. Electroencephalogr Clin Neurophysiol 89:22-28.

Benecke R, Rothwell JC, Dick JPR, Day BL, Marsden CD (1987) Disturbance of sequential movements in patients with Parkinson's disease. Brain 110:361-379.

Berns GS, Sejnowski TJ (1998) A computational model of how the basal ganglia produce sequences. J Cogn Neurosci 10:108-121.

Bhutani N, Sureshbabu R, Farooqui AA, Behari M, Goyal V, Murthy A (2013) Queuing of concurrent movement plans by basal ganglia. J Neurosci 33:9985-9997.

Bloem BR, Grimbergen YAM, van Dijk JG, Munneke M (2006) The "posture second" strategy: a review of wrong priorities in Parkinson's disease. J Neurol Sci 248:196-204.

Braak H, Tredici KD, Rüb U, de Vos RAI, Jansen Steur ENH, Braak E (2003) Staging of brain pathology related to sporadic Parkinson's disease. Neurobiol Aging 24:197-211.

Cools AR, van den Bercken JH, Horstink MW, van Spaendonck KP, Berger HJ (1984) Cognitive and motor shifting aptitude disorder in Parkinson's disease. J Neurol Neurosurg Psychiatry 47:443-453.

Desmurget M, Grafton ST, Vindras P, Gréa H, Turner RS (2003) Basal ganglia network mediates the control of movement amplitude. Exp Brain Res 153:197-209.

Desmurget M, Gaveau V, Vindras P, Turner RS, Broussolle E, Thobois S (2004) On-line motor control in patients with Parkinson's disease. Brain 127:1755-1773.

Díaz-Hernández E, Contreras-López R, Sánchez-Fuentes A, Rodríguez-Sibrían L, Ramírez-Jarquín JO, Tecuapetla F (2018) The thalamostriatal projections contribute to the initiation and execution of a sequence of movements. Neuron 100:739-752.e5.

Dixon P, Glover S (2009) Perseveration and contrast effects in grasping. Neuropsychologia 47:1578-1584.

Downes JJ, Sharp HM, Costall BM, Sagar HJ, Howe J (1993) Alternating fluency in Parkinson's disease. An evaluation of the attentional control theory of cognitive impairment. Brain 116:887902.

Garr E (2019) Contributions of the basal ganglia to action sequence learning and performance. Neurosci Biobehav Rev 107:279-295.

Gentilucci M, Negrotti A (1999) The control of an action in Parkinson's disease. Exp Brain Res 129:269-277.

Graybiel AM (1998) The basal ganglia and chunking of action repertoires. Neurobiol Learn Mem 70:119-136.

Haaxma CA, Bloem BR, Overeem S, Borm GF, Horstink MWIM (2010) Timed motor tests can detect subtle motor dysfunction in early Parkinson's disease. Mov Disord 25:1150-1156.
Halford GS, Wilson WH, Phillips S (1998) Processing capacity defined by relational complexity: implications for comparative, developmental, and cognitive psychology. Behav Brain Sci 21:803-831.

Hayes AE, Davidson MC, Keele SW, Rafal RD (1998) Toward a functional analysis of the basal ganglia. J Cogn Neurosci 10:178-198.

Helmich RC, Aarts E, de Lange FP, Bloem BR, Toni I (2009) Increased dependence of action selection on recent motor history in Parkinson's disease. J Neurosci 29:6105-6113.

Hesse C, De Grave DDJ, Franz VH, Brenner E, Smeets JBJ (2008) Planning movements well in advance. Cogn Neuropsychol 25:985-995.

Horak FB, Frank J, Nutt J (1996) Effects of dopamine on postural control in parkinsonian subjects: scaling, set, and tone. J Neurophysiol 75:2380-2396.

Humphries MD, Stewart RD, Gurney KN (2006) A physiologically plausible model of action selection and oscillatory activity in the basal ganglia. J Neurosci 26:12921-12942.

Jáidar O, Carrillo-Reid L, Hernández A, Drucker-Colín R, Bargas J, Hernández-Cruz A (2010) Dynamics of the Parkinsonian striatal microcircuit: entrainment into a dominant network state. J Neurosci 30:11326-11336.

Jax SA, Rosenbaum DA (2009) Hand path priming in manual obstacle avoidance: rapid decay of dorsal stream information. Neuropsychologia 47:1573-1577.

Jax SA, Rosenbaum DA (2007) Hand path priming in manual obstacle avoidance: evidence that the dorsal stream does not only control visually guided actions in real time. J Exp Psychol Hum Percept Perform 33:425-441.

Johnson KA, Cunnington R, Bradshaw JL, Phillips JG, lansek R, Rogers MA (1998) Bimanual co-ordination in Parkinson's disease. Brain 121:743-753.

Kish SJ, Shannak K, Hornykiewicz O (1988) Uneven pattern of dopamine loss in the striatum of patients with idiopathic Parkinson's disease. N Engl J Med 318:876-880.

Kordower JH, Olanow CW, Dodiya HB, Chu Y, Beach TG, Adler CH, Halliday GM, Bartus RT (2013) Disease duration and the integrity of the nigrostriatal system in Parkinson's disease. Brain 136:24192431.

Kornysheva K, Diedrichsen J (2014) Human premotor areas parse sequences into their spatial and temporal features. Elife 3:e03043.

Langston JW, Widner H, Goetz CG, Brooks D, Fahn S, Freeman T, Watts $R$ (1992) Core assessment program for intracerebral transplantations (CAPIT). Mov Disord 7:2-13.

Mazzoni P, Hristova A, Krakauer JW (2007) Why don't we move faster? Parkinson's disease, movement vigor, and implicit motivation. J Neurosci 27:7105-7116.

Redgrave P, Gurney K (2006) The short-latency dopamine signal: a role in discovering novel actions? Nat Rev Neurosci 7:967-975.

Redgrave P, Prescott TJ, Gurney K (1999) The basal ganglia: a vertebrate solution to the selection problem? Neuroscience 89:10091023.

Redgrave P, Rodriguez M, Smith Y, Rodriguez-Oroz MC, Lehericy S, Bergman H, Agid Y, DeLong MR, Obeso JA (2010) Goal-directed and habitual control in the basal ganglia: implications for Parkinson's disease. Nat Rev Neurosci 11:760-772.

Rosenbaum DA, Cohen RG, Jax SA, Weiss DJ, van der Wel R (2007) The problem of serial order in behavior: Lashley's legacy. Hum Mov Sci 26:525-554.

Shadmehr R, Krakauer JW (2008) A computational neuroanatomy for motor control. Exp Brain Res 185:359-381.

Tang R, Whitwell RL, Goodale MA (2015) The influence of visual feedback from the recent past on the programming of grip aperture is grasp-specific, shared between hands, and mediated by sensorimotor memory not task set. Cognition 138:49-63.

Taverna S, Ilijic E, Surmeier DJ (2008) Recurrent collateral connections of striatal medium spiny neurons are disrupted in models of Parkinson's disease. J Neurosci 28:5504-5512.

Tremblay P-L, Bedard M-A, Langlois D, Blanchet PJ, Lemay M, Parent $M(2010)$ Movement chunking during sequence learning is 
a dopamine-dependant process: a study conducted in Parkinson's disease. Exp Brain Res 205:375-385.

van der Wel RPRD, Fleckenstein RM, Jax SA, Rosenbaum DA (2007)

Hand path priming in manual obstacle avoidance: evidence for abstract spatiotemporal forms in human motor control. J Exp Psychol Hum Percept Perform 33:1117-1126.
Wu T, Wang L, Hallett M, Li K, Chan P (2010) Neural correlates of bimanual anti-phase and in-phase movements in Parkinson's disease. Brain 133:2394-2409.

Wymbs NF, Bassett DS, Mucha PJ, Porter MA, Grafton ST (2012) Differential recruitment of the sensorimotor putamen and frontoparietal cortex during motor chunking in humans. Neuron 74:936-946. 